\title{
DESARROLLO REGIONAL, ELECTRIFICACIÓN Y REORGANIZACIÓN SOCIOESPACIAL EN VALLE DE BRAVO, MÉXICO
}

\author{
Nancy Sierra López \\ nansysie@yahoo.com.mx
}

UAEM

Alejandro Tonatiuh Romero Contreras lautona@aol.com Centro Interamericano de Recursos del Agua (CIRA) y UAEM

Lilia Zizumbo Villarreal lzv04@yahoo.com

UAEM

\section{RESUMEN}

En México, la planificación de cuencas hidrográficas propuso, entre otras cosas, la administración y gestión del agua a través de la realización de obras hidroeléctricas con la finalidad principal de proporcionar electricidad a la creciente industria nacional. El Proyecto Hidroeléctrico Miguel Alemán, ubicado al sur del estado de México, ilustra las consecuencias de esta forma de desarrollo, razón por la cual el presente artículo tiene como objetivo identificar los cambios ambientales derivados de la construcción de una de las seis presas que lo conforman, la de Valle de Bravo. Así, los sutiles lazos sociales que permitían a la comunidad actuar por el interés colectivo fueron socavados por nuevas relaciones. De igual forma, los recursos como la tierra o el agua se han convertido en mercancías y el acceso a ellos está determinado no por las relaciones sociales, sino por el mercado.

Palabras clave: desarrollo regional, presas hidroeléctricas, cambios socioespaciales, Valle de Bravo. 
ABSTRACT

In Mexico, watershed-based planning included water administration and management by means of constructing hydroelectric plants. The main aim was to supply electric power to a fast growing national industry. The Miguel Alemán Hydroelectric Project, located in the south of the State of Mexico, illustrates the consequences of this form of development. This article thus aims to identify environmental changes derived from the construction of one of the six dams which make up this project, the one in Valle de Bravo. Thus, the subtle social bonds which enabled the community to act on behalf of collective interests have been undermined by new social relations. Likewise, resources such as soil and water have become commercial goods the access to which is determined not by social relations, but by the market.

Key words: regional development, hydroelectric dams, socio-spatial changes, Valle de Bravo. 


\section{INTRODUCCIÓN}

El proceso de desarrollo en México ha sido objeto del pensamiento científico desde hace ya mucho tiempo y establece una serie de relaciones que trascienden lo económico, lo social y lo político. De esta forma, algunos sostienen que el desarrollo es concebido como un puro fenómeno de crecimiento económico, mientras que otros lo consideran, además, como un proceso de cambios estructurales y funcionales que modifican la totalidad de la sociedad, reflejándose especialmente en su sistema de clases (Preston 1999).

Hacia finales de 1920, el Estado moderno mexicano propuso guiar de forma planificada el desarrollo de las regiones nacionales, surgiendo así diversos mecanismos para alcanzarlo, algunos de los cuales llevaron implícitas decisiones estratégicas de carácter territorial y alteraron el curso histórico de una sociedad determinando su estructura y sus características futuras. Desde esta perspectiva, las políticas de planeación regional son incorporadas como instrumentos para alcanzar el desarrollo capitalista, pues contienen un creciente poder predictivo que les permite controlar y modificar deliberada y conscientemente tanto el ambiente como las instituciones y los procesos sociales, estimulando la aparición y localización de ciertas actividades humanas en el paisaje (Palerm 1993).

Bajo este contexto, la construcción de grandes presas constituyó parte de las políticas de desarrollo llevadas a cabo en México, en razón de las crecientes demandas energéticas derivadas del modelo industrializador así como de las llamadas políticas de desarrollo regional orientadas a promover el crecimiento económico de las regiones consideradas marginales. Las presas fueron percibidas como la estrategia adecuada para superar los desequilibrios estructurales interregionales y homogeneizar el tan anhelado desarrollo nacional. Sin embargo, los resultados obtenidos son contradictorios, pues mientras que algunos de sus logros, como la generación de energía eléctrica y el riego permanente, promueven el desarrollo de ciertos grupos y regiones; los sectores más pobres de la población (indígenas, campesinado tradicional, marginados urbanos), son quienes ceden 
sus tierras para dichas obras y quienes menos o ningún beneficio obtienen de ellas (Sallenave 1992: 41). Causan entonces una serie de daños en las regiones receptoras que se manifiestan en graves consecuencias tanto socioculturales como ambientales.

El sistema hidroeléctrico Miguel Alemán, ubicado en el municipio de Valle de Bravo al sur del estado de México, ilustra las consecuencias de esta forma de desarrollo, pues al establecerse la industria en la Ciudad de México y en Toluca, uno de los principales requerimientos fue la energía eléctrica, la cual resultaba insuficiente, razón por la cual se creyó conveniente construir una serie de presas escalonadas para producir la electricidad demandada por esta actividad económica.

El objetivo principal de este artículo consiste en identificar los cambios generados por la construcción de la presa en Valle de Bravo, la cual formó parte del sistema hidroeléctrico Miguel Alemán y fue sin duda uno de los principales sucesos que modificó tanto el sistema natural como el sistema económico y socioespacial de este municipio. Asimismo, el proceso de reforma agraria que se desarrolló durante la década de 1930 también afectó de forma significativa la estructura espacial del lugar. Cabe señalar que en este documento sólo se aborda el periodo que va de 1930, fecha aproximada en que se inicia la construcción de la obra, hasta 1980, año en que se consolida una nueva actividad económica en el municipio, el turismo residencial, y también se transita a un nuevo modelo de abordar el espacio, el ordenamiento territorial.

En cuanto al proceso teórico-metodológico, la construcción teórica retoma el análisis de la relación que existe entre la teoría del desarrollo y los modelos de planeación territorial, y su influencia en la electrificación del país por medio de la construcción de sistemas hidroeléctricos. En cuanto al proceso metodológico, éste se llevó a cabo a través del análisis diacrónico de documentos oficiales, fotografías, mapas y croquis encontrados en los archivos históricos nacional, estatal y municipal. Se aplicaron también entrevistas a informantes clave y se procedió a la observación directa y a la comparación entre la información cartográfica y las actuales condiciones del territorio. De esta forma, «el trabajo de campo resultó imprescindible en la zona de estudio, no 
sólo en el reconocimiento ambiental, sino fundamentalmente en la interacción con la población» (Romero 2000:37).

\section{LA PLANEACIÓN DEL DESARROLLO}

El proceso de expansión económica a nivel mundial, generado por la Revolución industrial, se paralizó hacia finales de 1920 en el marco de la crisis económica internacional y del agotamiento del modelo orientado a la exportación que había prevalecido desde la década de 1880. Asimismo, la creciente ideología socialista encontraba en América Latina y Asia un campo fértil en función de las condiciones económicas y políticas producto de la posguerra. Para evitar la expansión de la influencia socialista, la comunidad norteamericana se interesó por generar propuestas que impulsaran el crecimiento, mas no el desarrollo, de las nuevas economías, surgiendo así la escuela de la modernización. De acuerdo con Preston (1999), este esquema indica que para alcanzar el desarrollo debería producirse una transición de una sociedad tradicional a una sociedad moderna, lo cual se lograría a través de la evolución gradual de las formas de producción hasta lograr que los países subdesarrollados (sociedades tradicionales) consiguieran el avance tecnológico necesario para transformarse en sociedades industrializadas. De esta forma, bajo la influencia de este modelo de desarrollo denominado keynesiano, el Estado sostuvo que la principal causa del atraso y la pobreza económica del país consistía en el predominio casi absoluto de las actividades primarias y en la debilidad o ausencia de las actividades secundarias, por lo cual se planteó la necesidad nacional de la industrialización. Sin embargo, este planteamiento se pone en duda como argumentaremos en el presente artículo.

Los proyectos dirigidos a la modernización se impulsaron bajo la convicción de que era factible guiar el desarrollo de las sociedades nacionales en forma planificada en función de objetivos previamente establecidos. De acuerdo con Viqueira (2001), uno de los proyectos de desarrollo que se implementaron tomando en cuenta la planeación del territorio a escala regional y que sirvió de modelo para desarrollar otras cuencas fluviales fue el llevado a cabo en Estados Unidos durante la administración del 
presidente Roosevelt en la cuenca del río Tennessee. Su finalidad era contrarrestar los efectos de la gran depresión, sin embargo, aunque se reanimaron ciertos sectores de la economía, en especial el sector industrial, también se agudizaron las diferencias de desarrollo entre ciertas área. Para reducir estas diferencias, el gobierno de Roosevelt implementó el Tennessee Valley, como parte de la política económica y social planteada en 1933 en el llamado New Deal.

El territorio delimitado para poner en marcha el proyecto de desarrollo fue un área geográfica configurada por una cuenca fluvial que pertenecía a cinco estados diferentes. El proyecto consistía en una serie de grandes obras hidráulicas para regularizar el curso del río Tennessee y al mismo tiempo aprovechar las presas y las caídas de agua para instalar turbinas que produjeran electricidad. La energía producida a bajo precio se destinó a electrificar el área rural y a crear una infraestructura para el desarrollo de la industria. Se transformaron entonces las plantaciones de algodón en granjas agrícolas mixtas y se procedió a redistribuir el terreno, de tal manera que se pasara del sistema de aparcería prexistente a un sistema de propiedad privada. Tenemos aquí un ejemplo de un proyecto de desarrollo integral en una región específica: Tennessee Valley Authority (TVA), que va desde la regularización del curso de los ríos a la industrialización y a la transformación de la agricultura. El resultado del proyecto se consideró a nivel internacional como un ejemplo exitoso de trasformación de una región deprimida en una región próspera (Viqueira 2001: 21).

El éxito de este proyecto sirvió de modelo para intentar desarrollar otras cuencas fluviales en América Latina. Sin embargo, los resultados distan mucho de los obtenidos por el TVA debido en gran parte a que la construcción de las presas hidroeléctricas tenía como objetivo favorecer a ciertos sectores económicos, dejando de lado el desarrollo de la región. Esto hace evidente que la planificación, al ser construida sobre juicios de valor, condiciona la toma de decisiones e incide en los resultados obtenidos. En consecuencia, la planificación es puesta al servicio de unos propósitos y de ciertas finalidades, expresados en lo que suele llamarse una política pública. En este sentido Palerm (2003) señala que: 
La planeación es un esfuerzo y una tentativa de racionalizar según finalidades, es decir, de ordenar y disponer la conducta de las comunidades humanas de acuerdo a ciertos propósitos que se persiguen, a ciertos fines que quieren conseguirse...es por ello que lo que realmente distingue y caracteriza a la planificación no es... aquello que se planifica, sino el carácter y la naturaleza de su acción. En consecuencia al discutir la planificación moderna debemos ocuparnos no sólo de sus técnicas, sino también de los propósitos que deben guiarla y de sus medios de ejecución, pues éstos le conceden por una parte su creciente poder predictivo y por otra su creciente capacidad humana de controlar y modificar deliberada y conscientemente tanto el medio ambiente como las instituciones y los procesos sociales (Palerm, 2003: 16).

De esta forma, ya desde la década de 1930, la planeación regional en México, considera la administración y gestión del agua, a través de la construcción de presas hidroeléctricas, como un proyecto a partir del cual se desarrollará principalmente el sector industrial y se podrá alcanzar de esta forma la modernización.

\section{ORGANIZACIÓN SOCIOESPACIAL DE VALLE DE BRAVO: UNA VISIÓN RETROSPECTIVA}

Antes de 1930, Valle de Bravo estuvo casi aislado del resto del estado de México debido a las escasas vías de comunicación, a su situación geográfica y al relieve accidentado de su superficie territorial. Sin embargo, su ubicación geográfica lo convertía en una zona de contacto entre las culturas del altiplano mexicano y las vecinas culturas de tierra caliente de los estados costeros de Guerrero y Michoacán hacia el occidente de México (ver mapa 1), constituyéndose como lugar de intercambio comercial, lo que acentuó el valor estratégico de la región desde el punto de vista económico (Ordenamiento Ecológico 2002). Esta ubicación privilegiada fue articulada por la arriería y caminos de herradura cuyo centro se ubicó en la población de Valle de Bravo, donde llegaban y partían recuas cargadas de productos hasta de 500 animales de carga (Chacón 2004: 123).

Culturalmente, la llegada de los franciscanos en el siglo XVI a la subcuenca Valle de 
Bravo-Amanalco (CVB-A) permitió, por una parte, la construcción de grandes templos y haciendas y, por otra, fortaleció la organización socioespacial basada en la apropiación y el uso de recursos naturales como la tierra y el agua; de ahí que su economía estuvo fundamentada en la agricultura de riego intensivo. La zona principal donde se desarrolló esta actividad fue llamada «El Plan» (ver foto 1), que era una extensión plana de 822.53 has. Se encontraba ubicada en la cabecera municipal y, dadas las características geomorfológicas, hídricas, geológicas y climáticas existentes en la región, contaba con suelos fértiles, propicios para el desarrollo de la agricultura de riego y de temporal.

Mapa 1. Localización geográfica

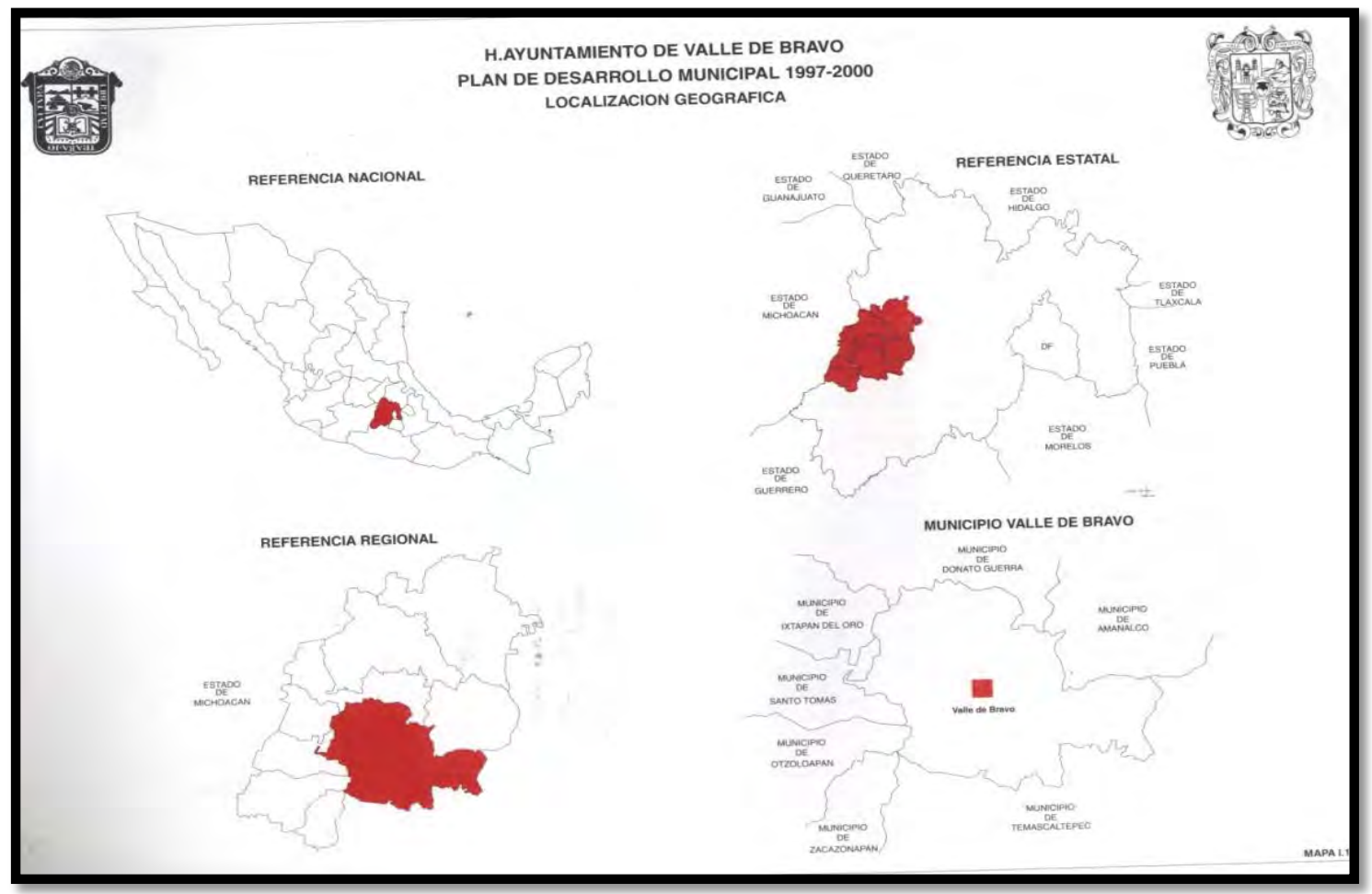

Fuente: Plan de Desarrollo Municipal. 1997-2000

En este sentido, algunos de los habitantes del lugar cosechaban productos de ciclo anual como maíz, cebada, alpiste, haba y trigo. También se cultivaba frijol, chícharo, calabaza y productos como cilantro, lechuga, cebolla, tomate, jitomate y rábanos, entre los más relevantes, empleándose el sistema de riego. En cuanto a frutales, se obtenían productos 
como lima, naranja, limón, granada, ciruelos y aguacates. De acuerdo con algunas versiones de los habitantes entrevistados, los pobladores que poseían terrenos en esta zona de riego y que se dedicaban a la agricultura también practicaban la ganadería mayor y menor, lo cual les permitió aprovechar los esquilmos como forrajes que sobraban de las cosechas (Bernal 2011). Los productos obtenidos eran utilizados para el autoconsumo y para la venta en las comunidades aledañas, pero principalmente eran transportados al Distrito Federal y a la ciudad de Toluca, que eran los principales centros de comercio.

Foto 1: “El Plan”, lugar donde se ubica la presa Valle de Bravo.

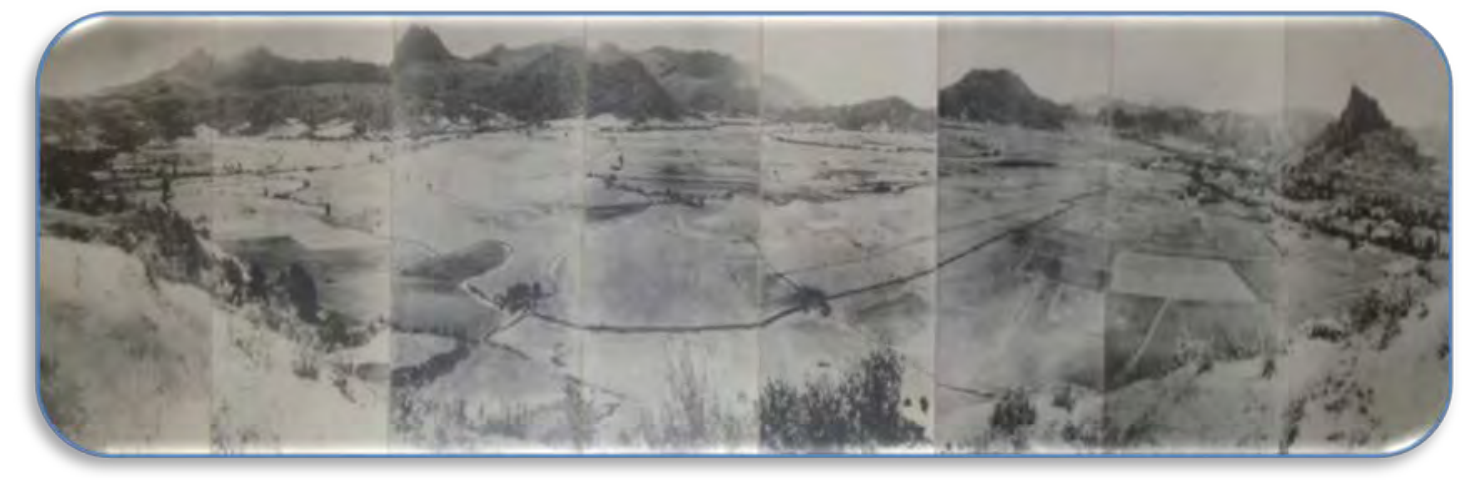

Fuente: Archivo fotográfico de Valle de Bravo.

Algunos otros vallesanos se dedicaban a la alfarería, elaborando productos como ollas, jarros, tazas y cazuelas, para lo cual cada familia se especializaba en la producción de alguna de estas mercancías. La mayoría de los materiales utilizados eran extraídos del mismo municipio. El barro y los tizates (tierra blanca que se mezcla con la «greta» u óxido de plomo para el vidriado de los productos) eran obtenidos de lugares cercanos. En cuanto a la leña, que se utilizaba para cocer las lozas, se traía de las zonas boscosas inmediatas. Sólo el cobre o la «greta», que se empleaban para dar el brillo final a los productos, eran traídos de Toluca o del Distrito Federal. Algunos entrevistados señalaron que los productos obtenidos se llevaban a vender a la ciudad de Toluca, a Tenancingo y a otros pueblos cercanos. Sin embargo, la gran mayoría prefería venderlos con los acaparadores, quienes cada ocho días llevaban un camión lleno de productos a la ciudad de Toluca. 
Por otra parte, los habitantes del centro de la cabecera se dedicaban principalmente al comercio y a la explotación de recursos forestales de los que se obtenía leña, carbón y resina que en su mayoría se vendían a los habitantes de la misma localidad. Aunado a esto, también se dedicaban al tejido de rebozos y a la fabricación de arreos para las bestias de carga. Fue así como los habitantes de Valle de Bravo aprovecharon sus recursos naturales consiguiendo desarrollar actividades de subsistencia y de beneficio.

Debido a la diversificación de actividades productivas, la cuenca Valle de BravoAmanalco fue considerada como una región económica importante hasta el periodo revolucionario, pues la región era controlada por los revolucionarios para evitar el flujo de mercancías hacia la Ciudad de México (Chacón 2004: 123).

Este suceso transformó las condiciones del municipio, pues al dejar de percibir los ingresos provenientes del comercio y de los servicios generados por las personas provenientes de 'tierra caliente', los habitantes de Valle de Bravo emigraron hacia Morelia, Toluca y la Ciudad de México en busca de mejores fuentes de ingresos. Cabe señalar que este suceso sólo afectó temporalmente la estructura social y económica del municipio, ya que el desarrollo de las actividades agrícolas permitió su recuperación gradual.

Sin embargo, en la década de 1930, el Estado mexicano implementó un modelo de desarrollo basado en la industrialización. Este hecho generó un aumento en la demanda de energía eléctrica, por lo que se implementó una serie de proyectos hidroeléctricos, los cuales se ubicaron en zonas con diversas pendientes que contaban con un abundante recurso hídrico. En el caso de Valle de Bravo, con la construcción de las presas los recursos naturales como la tierra, el bosque y principalmente el agua, dejaron de ser usados por los campesinos y pasaron a manos del Estado, quien les dio un uso diferente. Asimismo, se realizaron una serie de expropiaciones en la zona conocida como «El Plan», lo cual afectó seriamente a los habitantes del lugar que durante mucho tiempo habían subsistido de actividades derivadas del sector primario. 


\section{EL SISTEMA HIDROELÉCTRICO MIGUEL ALEMÁN}

En 1917 el gobierno federal asumió el dominio total de los recursos hidráulicos, elevándolos a rango de propiedad nacional para un mejor y mayor control en la generación de electricidad, la cual estaba controlada en un $97 \%$ por capital extranjero (Navarrete 2008: 99). En 1933 se planteó la discusión de los problemas relacionados con las industrias generadoras de energía eléctrica que hasta ese momento se encontraban en manos de tres monopolios: The Mexican Light and Power Company Limited y subsidiarias, la Nueva Compañía Eléctrica de Chapala y compañías afiliadas, y la American and Foreign Power Company. Asimismo, se envió la iniciativa de ley para crear la Comisión Federal de Electricidad (CFE), la cual se encargaría de realizar a nivel nacional las obras hidráulicas que permitieran la construcción de vasos de almacenamiento (presas) para la generación de energía eléctrica.

En este sentido, el modelo de desarrollo adoptado señalaba que la electricidad era un artículo necesario para el desarrollo de la industria dado el crecimiento de la demanda y la imposibilidad de abastecimiento por la empresa que la suministraba en el Distrito Federal. Derivado de este proceso, se realizaron algunas obras de electrificación en estados como Guerrero, Michoacán y Oaxaca, entre otros. Asimismo, se continuaron los estudios iniciados por la Cía. de Fuerza del Suroeste de México, filial de la Mexican Light and Power Co., cuyos proyectos sirvieron de base para iniciar los trabajos de construcción del sistema hidroeléctrico de Ixtapantongo en Valle de Bravo. Fue en esta área perteneciente a la cuenca del Balsas donde las características geográficas e hidrológicas permitieron establecer el proyecto Ixtapantongo, que más tarde cambiaría su nombre a «Miguel Alemán» y que posteriormente ampliaría su aprovechamiento hasta establecer seis plantas generadoras de electricidad. En 1938, el Estado transfirió la responsabilidad de este proyecto a la CFE.

El sistema hidroeléctrico Miguel Alemán estaba ubicado al suroeste de la ciudad de Toluca y se encontraba integrado por seis plantas escalonadas que abarcaban unos 60 
km de longitud (Ver croquis 1 ).

Croquis 1. Sistema hidroeléctrico «Miguel Alemán», Estado de México.

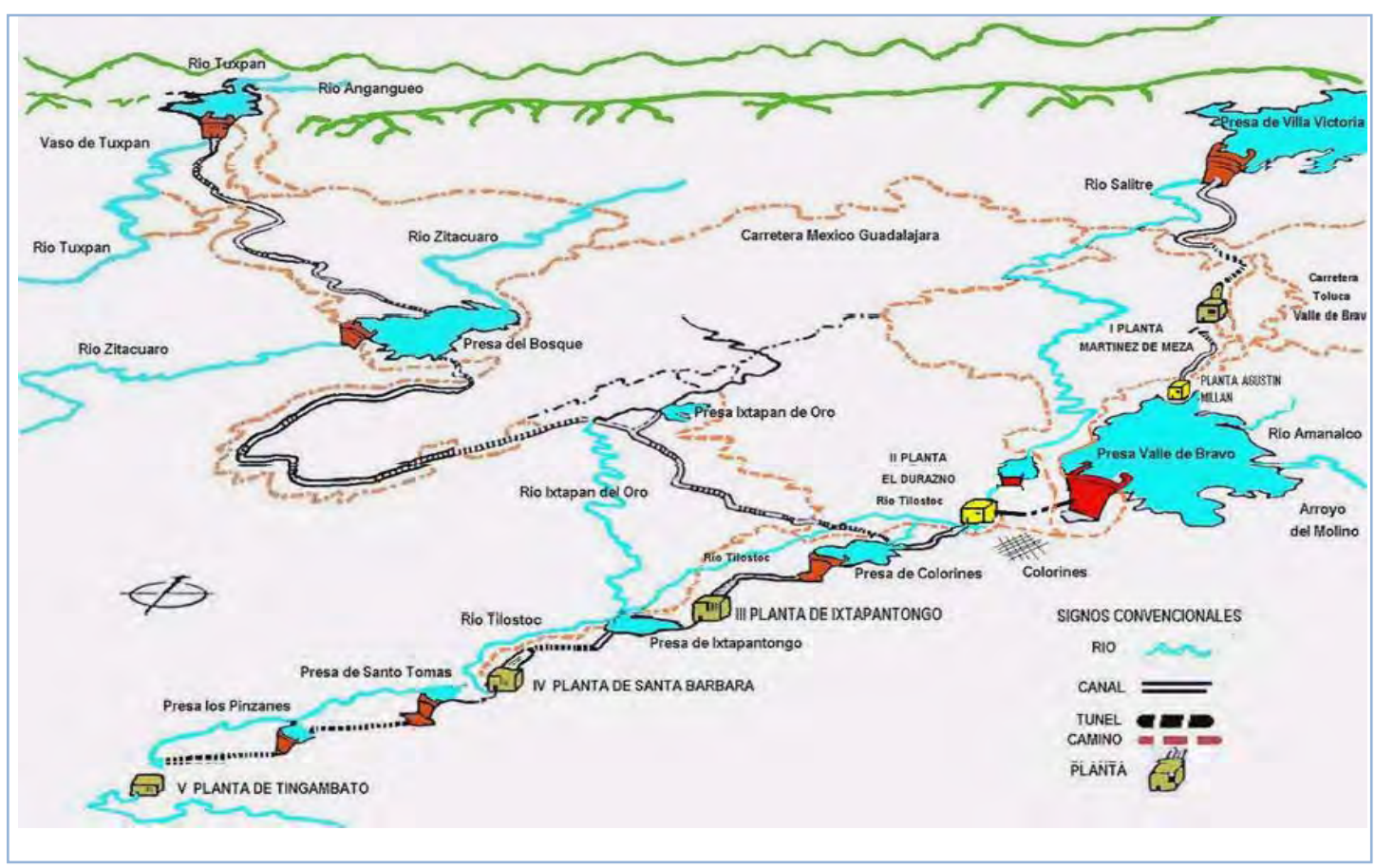

Fuente: El sistema Cutzamala, en www.conagua.gob.mx [consulta: diciembre de 2010].

Sus plantas proporcionaban energía eléctrica para satisfacer las necesidades de la Ciudad de México y lugares circunvecinos. Empezó a construirse en 1938, iniciándose los trabajos en la central denominada Ixtapantongo (por ocupar los terrenos de la hacienda del mismo nombre). En este sistema se aprovechaba el desnivel de las corrientes de los ríos San José Malacatepec, Valle de Bravo, Ixtapan del Oro, Salitre, Amanalco, San Juan, González y el Molino en el estado de México y, en el estado de Michoacán, de los ríos Tuxpán y Zitácuaro, todos afluentes de los ríos Tilostoc o Cutzamala. Para el aprovechamiento de dichos cuerpos de agua se construyeron las siguientes obras: 
Cuadro 1. Obras que formaron el sistema hidroeléctrico Miguel Alemán, Estado de México.

\begin{tabular}{|c|c|c|}
\hline OBRA & CARACTERÍSTICAS & $\begin{array}{c}\text { PLANTA } \\
\text { HIDROELÉCTRICA QUE } \\
\text { ALIMENTA }\end{array}$ \\
\hline Presa Villa Victoria & $\begin{array}{l}\text { Almacena las aguas provenientes del río } \\
\text { San José Malacatepec desde su nivel más } \\
\text { alto (2605 msnm). Sus aguas se } \\
\text { conducen y almacenan en la presa Valle } \\
\text { de Bravo. }\end{array}$ & $\begin{array}{l}\text { Ing. Héctor Martínez de Meza } \\
\text { Gral. Agustín Millán. }\end{array}$ \\
\hline Presa Valle de Bravo & $\begin{array}{l}\text { Tiene una altitud de } 1,830 \text { msnm y } \\
\text { almacena el } 50 \% \text { del agua del sistema. }\end{array}$ & \\
\hline Presa Tilostoc & $\begin{array}{l}\text { Ubicada en la confluencia de los ríos } \\
\text { Malacatepec y Tilostoc donde se vertían } \\
\text { las aguas de la presa de Valle de Bravo. } \\
\text { El objetivo principal de esta presa fue } \\
\text { regular y decantar las aguas de las } \\
\text { cuencas libres de Malatepec para } \\
\text { disminuir la acumulación de azolve y } \\
\text { continuar la conducción de agua de un } \\
\text { canal al siguiente. }\end{array}$ & El Durazno \\
\hline $\begin{array}{l}\text { Vaso regulador de } \\
\text { Colorines }\end{array}$ & Tiene una altitud de 1,586 msnm. & \\
\hline Presa de Ixtapantongo & & Santa Bárbara \\
\hline Presa de Santo Tomás & $\begin{array}{l}\text { Regulaba las aguas provenientes de las } \\
\text { presas anteriores. Sus aguas inundaron el } \\
\text { viejo pueblo del mismo nombre, } \\
\text { generando cambios ambientales. }\end{array}$ & \\
\hline Presa Pinzares & $\begin{array}{l}\text { Es la última presa del sistema y fue la } \\
\text { primera hidroeléctrica subterránea } \\
\text { construida en 1957. Es la de mayor } \\
\text { capacidad en toda la República } \\
\text { Mexicana. }\end{array}$ & Tingambato. \\
\hline
\end{tabular}

Fuente: Elaboración propia con base en información obtenida en Sallenave (1992).

LA CONSTRUCCIÓN DE LA PRESA VALLE DE BRAVO: CAMBIOS SOCIOESPACIALES, EXPROPIACIONES Y REUBICACIONES

En la región donde se construyó el sistema hidroeléctrico Miguel Alemán había varias 
haciendas entre las que destacan las de San Pedro Ixtapantongo, San Nicolás Tolentino, San Gabriel Metlaltepec, La Labor y Calderones, así como varios ranchos dependientes de las mismas como Santa Teresa Tiloxtoc o Cerro del Rosario, Las Trojes, El Cerrillo, Milpillas y Chila, entre otros. Su fraccionamiento inició cuando la Compañía de Fuerza Motriz del Suroeste de México compró alrededor de 224 has de la hacienda Ixtapantongo para establecer el campamento de Colorines y con ello dar inicio oficial a los trabajos del sistema hidroeléctrico Miguel Alemán. Posteriormente, con la aplicación de la reforma agraria, en 1937 se dotó de tierra a los habitantes que antes habían labrado tales tierras en calidad de peones. Ante este reacomodo agrario, se desarrollaron varias luchas internas entre propietarios de haciendas y ejidatarios, resultando beneficiados temporalmente algunos de los campesinos de Valle de Bravo.

Sin embargo, una vez que estuvo determinada la factibilidad para la construcción de la presa, y como producto de la reforma agraria, un nuevo cambio socioespacial tendría lugar: comenzaron a surgir los decretos de expropiación de los terrenos que serían ocupados por dicho cuerpo de agua, entre los que se encontraban los terrenos de las haciendas La Labor y El Cerrillo, El Zancudo, San Juan, San Lorenzo, San Antonio, y otros predios más pequeños.

De esta forma, el 19 de noviembre de 1946 la Secretaría de Economía Nacional publicó en el Diario Oficial de la Federación un decreto mediante el cual se expropiaban los terrenos comprendidos en la curva de embalse a la cota de 1,833 msnm, que serían inundados por el agua de la presa de Valle de Bravo. Según enuncia este decreto, la Comisión Federal de Electricidad (CFE) trataría con los productores rurales que resultarían afectados por la expropiación, procurando llegar a un arreglo económico y territorial. En este mismo documento se presenta el número de hectáreas que les serían expropiadas, así como la superficie total, que sería de 822-53-94 has aproximadamente.

La indemnización por los terrenos expropiados fue cubierta en efectivo por la Comisión Federal de Electricidad. Se depositó la suma en el Banco Nacional de Crédito Ejidal a nombre del Comisariado Ejidal del poblado de Valle de Bravo, pasando a formar parte 
del fondo común del ejido. Fue invertida en los términos del artículo 194, en relación con el 213 y el 214, todos del Código Agrario vigente en ese periodo, el cual establecía que:

las compensaciones por expropiación, deberían consistir de preferencia en terrenos de la misma calidad o equivalentes a los expropiados... cuando sean pagadas total o parcialmente en efectivo se invertirá en primer lugar, en la adquisición de terrenos de cultivo, para reponer los que hubieran sido tomados de las parcelas individuales, en segundo término para adquirir cualquiera otra clase de tierras que convenga al mejoramiento del ejido, y en tercero, para los fines indicados en el artículo 214 que establece obras de mejoramiento territorial, construcción de escuelas, obras de riego, servicios urbanos. O bien para la adquisición de maquinaria, animales de trabajo o de cría, aperos, semillas, etc. (Código Agrario 1942).

Según los registros existentes, la fecha en que quedó concluida la cortina de presa de Valle de Bravo fue el 17 de julio de 1947, dando inicio a la inundación de «El Plan». Algunas entrevistas con habitantes de Valle de Bravo que tuvieron la oportunidad de ver este proceso indican que, antes de que fuera cerrada la compuerta de la presa de forma definitiva, se hicieron algunas pruebas para verificar su resistencia. Por lo tanto, abrían y cerraban la compuerta para detectar filtraciones. Dicha situación duró bastante tiempo y alentó a los agricultores a continuar con sus actividades durante los diez años más que duraron los trabajos, a pesar de que en el año en que se cerró se les advirtió que no lo hicieran, pues sus cultivos se perderían con la inundación. Sin embargo, la mayoría comenta que, aun cuando la cortina de la presa fue cerrada de forma definitiva, el nivel de agua ascendió de forma muy lenta, por lo que algunos pobladores continuaron sembrando en las orillas que aún no habían sido alcanzadas por el agua.

En 1958 se registró un mayor índice de lluvias, lo cual ayudó a que la presa alcanzara su nivel máximo. Para ello también fue necesario reforestar una significativa cantidad de superficie cerril, creándose un vivero para la producción de plantas como jacarandas, eucaliptos, pinos y otros, con el objetivo de atraer las lluvias para el almacenamiento de agua en los vasos de la presa. Estos cambios en el aspecto natural derivaron en una serie 
de prohibiciones en las actividades silvoagrícolas practicadas dentro de la cuenca. De esta forma, al ser despojados de sus bienes de producción, una parte de la población nativa se trató de incorporar a las nuevas actividades económicas impuestas por la política de planeación territorial nacional, mientras que otra parte no tuvo opción mejor que emigrar y vender sus casas y tierras (Sierra 2011: 5).

Cabe señalar que no sólo los habitantes del municipio de Valle de Bravo resultaron afectados, ya que, al ser un proyecto de carácter regional, también se registraron expropiaciones en otros municipios. Tal es el caso de los ejidos de San Antonio de la Laguna y de San Francisco Mihualtepec, ambos pertenecientes al municipio de Donato Guerra. En el caso del primero, la superficie solicitada en expropiación por la CFE fue de 11.60 has de riego que formaron parte de la superficie inundada. En cuanto al ejido de San Francisco, se expropiaron 93.60 has de riego que también formaron parte del vaso de la presa de Valle de Bravo (Departamento Agrario 1954), «y aunque la presa no fue construida para esta gente, la vida en sus comunidades cambió drásticamente como resultado de la construcción de la misma» (McMahon 1973: 11). En el cuadro 2 se muestran las expropiaciones más relevantes realizadas por la CFE.

Cuadro 2. Expropiaciones hechas por la CFE para la construcción del sistema hidroeléctrico Miguel Alemán en el estado de México

\begin{tabular}{|c|c|c|c|}
\hline $\begin{array}{c}\text { Fecha de aparición en } \\
\text { el Diario Oficial de la } \\
\text { Federación }\end{array}$ & Territorio expropiado & $\begin{array}{c}\text { Tipo de tenencia } \\
\text { de la tierra }\end{array}$ & Cantidad \\
\hline 19 de noviembre de 1946 & $\begin{array}{c}\text { Cabecera municipal Valle de } \\
\text { Bravo }\end{array}$ & Ejido/propiedad & 822.53 has \\
\hline 30 de abril de 1954 & $\begin{array}{c}\text { Ejido de la Laguna. Donato } \\
\text { Guerra }\end{array}$ & Ejido & 11.60 has riego \\
\hline 30 de abril de 1954 & $\begin{array}{c}\text { Ejido de la Candelaria. Valle de } \\
\text { Bravo }\end{array}$ & Ejido & 96.78 has riego \\
\hline 30 de abril de 1954 & $\begin{array}{c}\text { Ejido de San Francisco } \\
\text { Mihualtepec. Donato Guerra }\end{array}$ & Ejido & $\begin{array}{c}241.80 \text { has } \\
224 \text { (riego) } \\
17.6 \text { (temporal) }\end{array}$ \\
\hline
\end{tabular}

Fuente: Elaboración propia con base en las publicaciones realizadas en el Diario Oficial de la Federación.

Por otra parte, la CFE también fue la encargada de valorar la situación de la población afectada, pues con la inundación los habitantes del lugar iban a perder sus tierras de 
cultivo y en el mejor de los casos serían indemnizados o reubicados en otros terrenos. Para compensar los daños que se iban a generar, la CFE propuso tres formas de solución:

1) A la población que solicitaba continuar con sus actividades agrícolas se les acondicionarían terrenos en lugares próximos que de alguna manera fueran equiparables a las pérdidas ocasionadas y que les permitieran la continuidad de sus labores económico-productivas.

2) A aquéllos que pretendían encauzar sus actividades hacia otras ramas como la artesanía y el comercio, se les proporcionaría orientación, fomento, organización y creación de nuevas industrias, en las cuales se daría cabida no sólo a los antiguos propietarios, sino también a los trabajadores de la región.

3) Finalmente, a los que no podían por propia cuenta atender directamente las industrias por crear, se les apoyaría para que pudieran trabajar como obreros dentro de las mismas.

Desafortunadamente, las soluciones mencionadas no se llevaron a cabo pues, según comentan los lugareños, hubo gente que por las circunstancias tuvo que entregar sus tierras a la CFE. Aunque los propietarios esperaban cierta cantidad de dinero como indemnización o buscaban su reubicación en tierras productivas de acuerdo con las negociaciones a las que se había llegado, sin embargo, conforme pasaba el tiempo y no obtenían ningún beneficio, comenzó a generarse un conflicto social que derivó en la dotación de terrenos pertenecientes a los municipios de San Martín Otzoloapan y zonas aledañas a la orilla de la presa. Dichos terrenos fueron designados por la Comisión Federal de Electricidad y las autoridades municipales y estatales (Archivo Municipal Valle de Bravo, 1940). Sin embargo, se encontraban en lugares alejados, de difícil acceso o con características inadecuadas para la práctica de actividades agrícolas, razón por la cual la gente prefirió venderlos. Esta situación dio origen a una nueva ocupación del territorio y a una serie de conflictos agrarios, los cuales se solucionaron con la resolución emitida en el estudio socioeconómico realizado por el Departamento Agrario del gobierno federal en los años 1945-1947, en el cual se establecían los límites 
definitivos entre la comunidad de la cabecera municipal y sus barrios, Santa María Ahuacatlan, Otumba y La Peña, con el ejido de Acatitlán. (Departamento Agrario 1947).

Bajo este contexto, es posible observar que las políticas territoriales han generado en Valle de Bravo cambios socioespaciales significativos que derivaron en una dinámica demográfica y socioeconómica manifestada en nuevos usos de suelo en el territorio (ver mapas 2, 3 y 4).

Mapa 2. Distribución del uso del suelo antes de la construcción de la presa (1930)

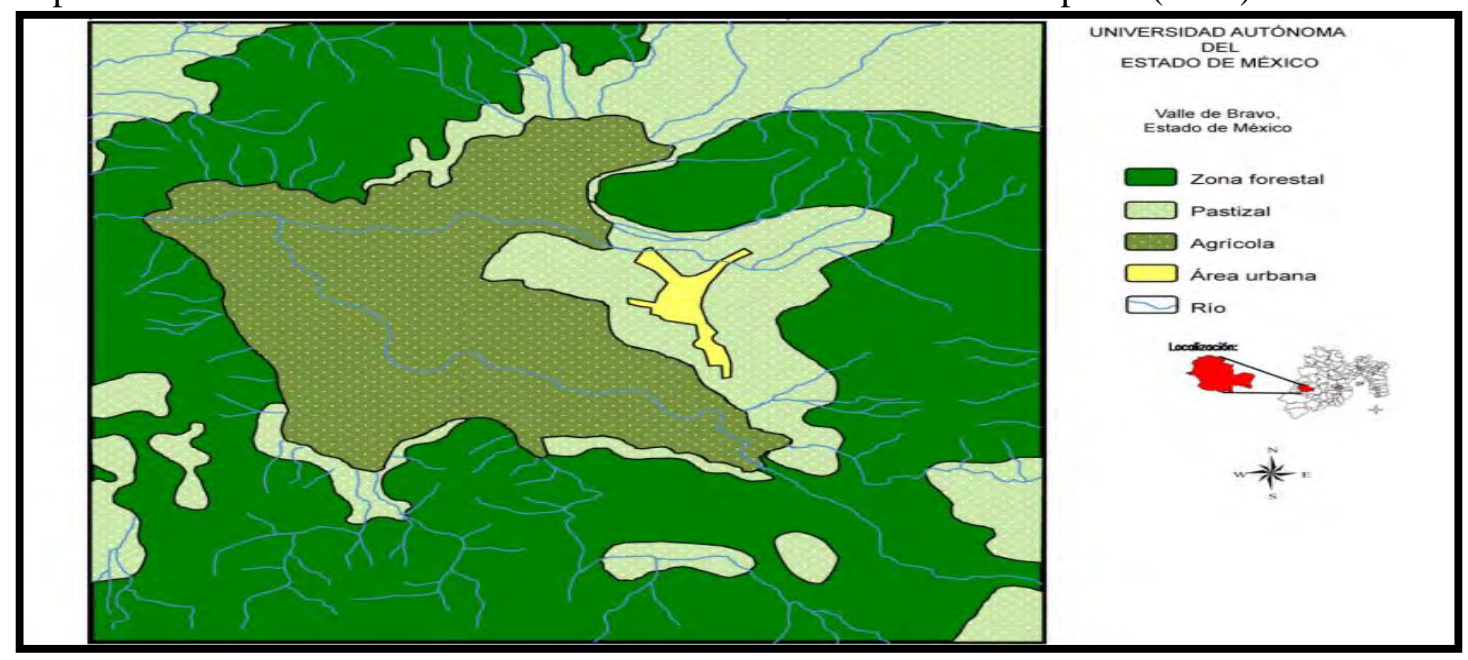

Fuente: Bernal, 2011 (escala 1: 50,000).

Mapa 3. Distribución del uso del suelo después de la construcción de la presa (1970)

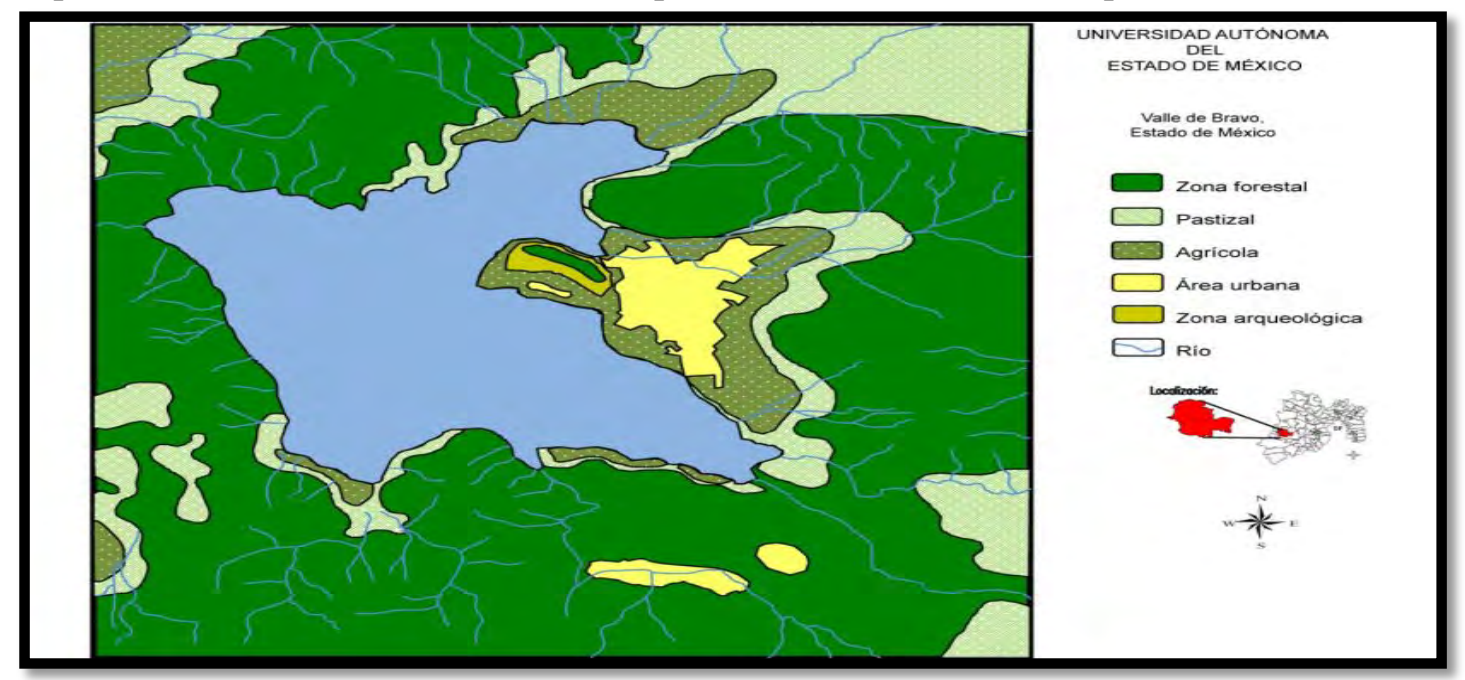

Fuente: Bernal, 2011 (escala 1:50,000). 
Mapa 4. Distribución del uso del suelo en la época actual (2010)

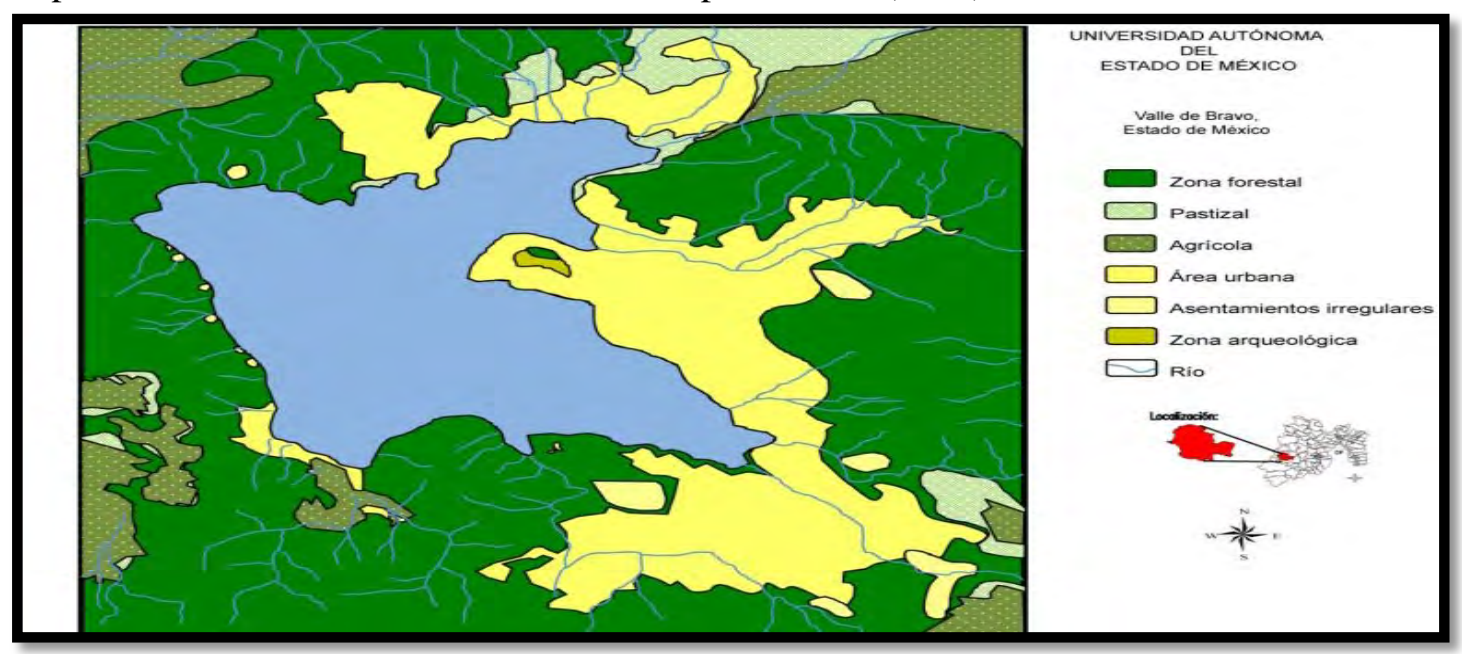

Fuente: Bernal, 2011 (escala 1:50,000)

\section{SURGIMIENTO DE COLORINES}

La construcción del sistema hidroeléctrico Miguel Alemán generó también cambios demográficos en la región, pues los trabajadores que se harían cargo de la construcción del mismo se ubicaron en zonas conocidas como campamentos. Entre ellos destacan el campamento «El Águila», ubicado en Santo Tomás de los Plátanos, y el campamento de «Colorines», ubicado en Valle de Bravo, ambos en el estado de México. Este último se estableció muy cerca de la ranchería de los Colorines, que en 1920 llegaba apenas a los 20 habitantes (7 mujeres y 13 hombres). Sin embargo, hacia 1937 arribaron los trabajadores de la CFE y aproximadamente 380 nuevos pobladores se establecieron en el lugar.

La actual Villa de Colorines albergó, desde sus inicios como campamento, a una considerable cantidad de trabajadores que aumentó rápidamente debido a las nuevas contrataciones laborales y a la migración de obreros pertenecientes a otros campamentos de la CFE ubicados en zonas aledañas. Tal es el caso del campamento del Águila, que para la década de 1950 contaba con un total de 1,076 habitantes que se encargaron de la construcción de la planta hidroeléctrica de Santa Bárbara. Este campamento tuvo una vida aproximada de cinco años, tiempo durante el cual se construyeron las rampas, presas y edificios que albergan las turbinas generadoras de 
electricidad. Sin embargo, en la década siguiente el campamento y sus habitantes habían desaparecido. Por testimonios orales sabemos que la mayoría de los pobladores se refugiaron en Colorines, otros regresaron a su lugar de origen y sólo unos cuantos permanecieron en Santo Tomás de los Plátanos.

Por estas razones, el número de habitantes en Colorines aumentó de manera significativa y al mismo tiempo las necesidades en cuanto a servicios básicos se volvieron apremiantes. Sin embargo, al no tener la categoría jurídica de pueblo, los apoyos recibidos eran mínimos y por ello surgió la inquietud de cambiar de categoría al campamento, para lo cual se formó la Comisión Pro-Pueblo de Colorines. Dicha comisión, junto con las autoridades delegacionales, llevó a cabo en 1962 un censo de población y vivienda, el cual dio como resultado un total de 4,520 habitantes (Navarrete 2008:52). Con base en este censo, solicitaron al gobernador del estado de México, Gustavo Baz Prada, que Colorines se elevara a pueblo, argumentando que por el número de habitantes había alcanzado esta categoría. Lo anterior se hizo oficial el $1^{\circ}$ de enero de 1963.

Gráfica 1. Cambios demográficos en Valle de Bravo (1910-2000)

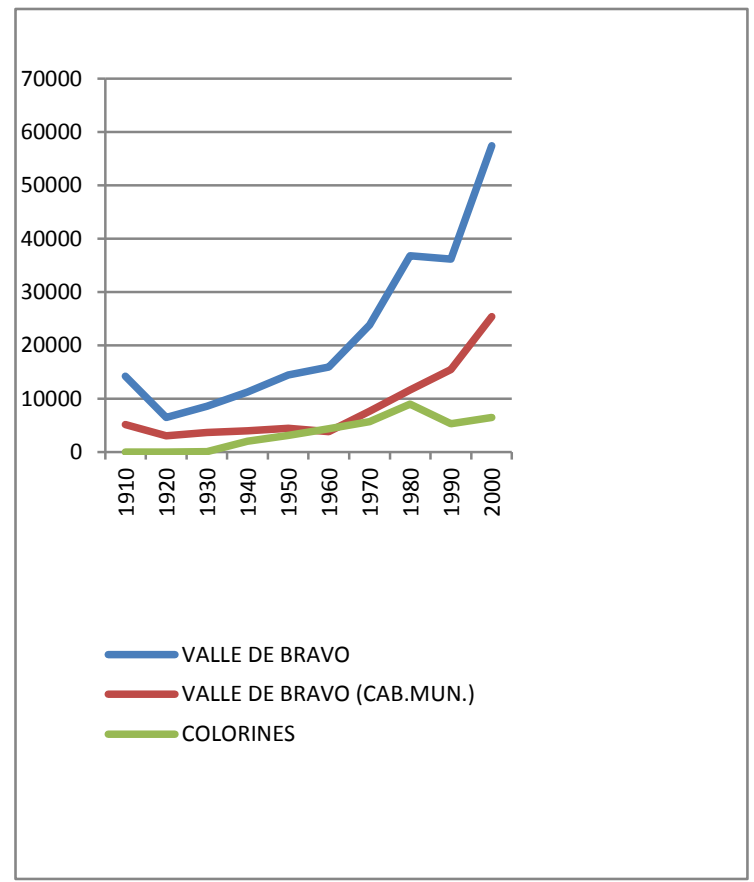

\begin{tabular}{|c|c|c|c|}
\hline & $\begin{array}{c}\text { Valle de } \\
\text { Bravo } \\
\text { (municipio) }\end{array}$ & $\begin{array}{c}\text { Valle de } \\
\text { Bravo } \\
\text { (cab. } \\
\text { municipal) }\end{array}$ & Colorines \\
\hline 1910 & 14,170 & 5,126 & - \\
\hline 1920 & 6,478 & 3,039 & 20 \\
\hline 1930 & 8,605 & 3,637 & 90 \\
\hline 1940 & 11,207 & 3,956 & 2,019 \\
\hline 1950 & 14,413 & 4,456 & 3,122 \\
\hline 1960 & 15,920 & 3,847 & 4,362 \\
\hline 1970 & 23,779 & 7,628 & 5,669 \\
\hline 1980 & 36,762 & 11,619 & 9,009 \\
\hline 1990 & 36,135 & 15,472 & 5,325 \\
\hline 2000 & 57,375 & 25,409 & 6,510 \\
\hline
\end{tabular}

Fuente: Elaboración propia, con base en los Censos de población y vivienda 1910-2000 (INEGI). 
A principios de 1980 se inauguró el arco de entrada al pueblo de Colorines. El acto protocolario estuvo a cargo del presidente de la República José López Portillo quien, aprovechando la ceremonia, dio a conocer un nuevo proyecto: el Sistema Cutzamala; pero fue tal la algarabía por la visita del ejecutivo federal que pocos tomaron en cuenta lo anunciado y el impacto que habría de causar. En las dos décadas posteriores, y tras el desmembramiento del sistema hidroeléctrico, el pueblo parecía no recuperarse por la sombra del desempleo y los ingresos, tanto del erario público como del bolsillo familiar, parecían ir en picada. Los resultados del censo de población de 1990 muestran una disminución importante de la población debido a tres éxodos de trabajadores de base transferidos por la CFE, quienes con sus familias abandonaron el pueblo para trasladarse a prestar sus servicios a otro lugar. Otros se jubilaron y regresaron a su lugar de origen, mientras que otros fallecieron.

De esta forma, el poblado que inició como un campamento, adquirió el 16 de noviembre de 2005 la categoría de Villa de Colorines. Asimismo, aumentó su territorio jurisdiccional, pues ahora se encuentra conformada por la propia Villa, Loma Bonita, El Durazno y el rancho los Colorines. Sin embargo, algunos de los protagonistas de esta nueva ocupación territorial se encuentran en situaciones desfavorables debido a la falta de empleo que generó para ellos el surgimiento del Sistema Cutzamala.

\section{CAMBIO EN LA ESTRUCTURA ECONÓMICA}

La dinámica económica que se presentó en el municipio después de la construcción de la presa Valle de Bravo se manifestó en el abandono paulatino de las actividades primarias, dando paso a la inserción de la población en actividades secundarias (sobre todo la industria de la construcción) y en actividades terciarias (a partir de 1980). Esta situación se debió en parte a que con la construcción de la presa se dotó a algunas familias sólo con una hectárea o menos de la mitad de ésta, razón por la cual sólo conseguían sembrar y cosechar apenas para el autoconsumo, por lo que todos los integrantes de la familia se vieron en la necesidad de buscar nuevos empleos dentro o 
fuera del municipio. Dichos cambios generaron en 1960 una migración de los habitantes que ocupaban la cabecera municipal de Valle de Bravo hacia la Ciudad de México, Michoacán y Guerrero (ver gráfica 1).

De este modo, la importancia de la agricultura para la población local fue desplazada y su lugar fue ocupado por actividades diferentes. Así, para el año 1960, y ya inundadas las tierras dedicadas antes a la agricultura, los habitantes del lugar comenzaron a dedicarse a la pesca y reproducción de diversas especies como charales, mojarras y lobinas, introduciendo posteriormente tilapia y carpa. Por esta razón, en 1963 se creó la Capitanía de Puerto cuya función principal era controlar las actividades y el tránsito de vehículos acuáticos que transportaban a los pobladores separados por el agua de la presa. Sin embargo, los vallesanos no se interesaron mucho en esta actividad por cuestiones culturales, pero también las restricciones que la misma Capitanía imponía para el uso y desarrollo de actividades en la presa impidieron que la pesca se convirtiera en una de las principales actividades económicas en el municipio.

Gráfica 2. Actividades económicas en Valle de Bravo (1930-2000)

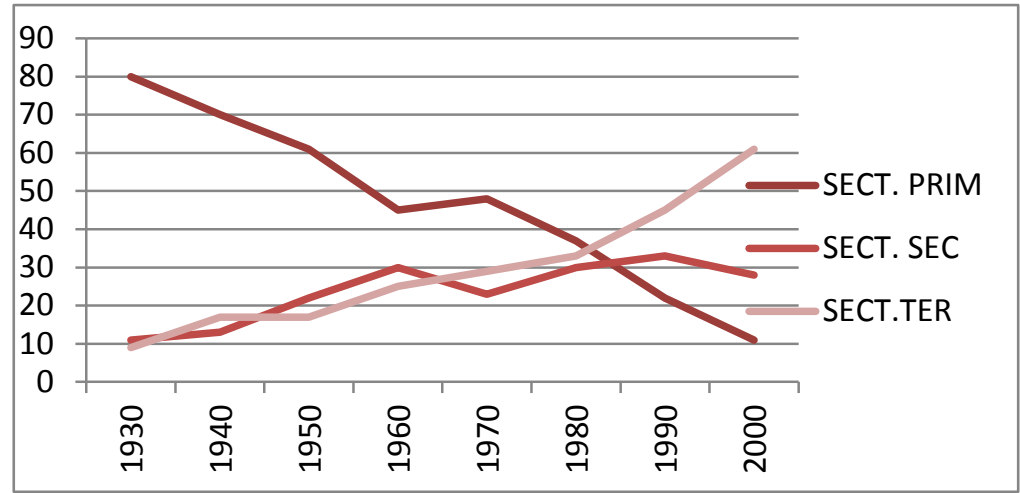

Fuente: Elaboración propia con base en los Censos de población y vivienda 1930-2010 (INEGI)

Al mismo tiempo, los terrenos aledaños a la presa estaban formados por bosques de pino encino pero, al estar prohibida su tala y debido al impulso de la reforestación promovido por las leyes forestales, los habitantes originarios del territorio no pudieron continuar su aprovechamiento ni dedicarse a las actividades silvoagrícolas que históricamente venían practicando, razón por la cual los vallesanos, dueños de esos espacios, decidieron, o mejor dicho fueron «obligados» por las políticas de protección al 
embalse, a venderlos. Fue la empresa pionera Propulsora de Valle de Bravo SA de CV, la que dio inicio a los trabajos de construcción del primer fraccionamiento en el municipio en la década de los cincuenta del siglo pasado, detonando por una parte el surgimiento del turismo residencial y por otra la industria de la construcción.

Así, la infraestructura creada en Valle de Bravo para la producción de electricidad conformó un paisaje atractivo caracterizado por un cuerpo de agua rodeado de zonas boscosas, lo que en una etapa posterior permitió la realización de actividades acuáticas que hasta entonces eran casi imposibles de practicar en el estado de México. Este tipo de política territorial de desarrollo facilitó la llegada de capitalistas fraccionadores ajenos al lugar, motivados también por los incentivos que el Estado otorgó durante ese periodo no sólo al establecimiento de la actividad industrial, sino también al de la actividad turística (García 1979: 18), creándose así nuevos destinos turísticos regionales como ocurrió en este municipio.

\section{CONCLUSIONES}

La expansión capitalista y la adopción del modelo de desarrollo regional basado en el establecimiento de la industria, generaron la necesidad de incrementar la producción de energía eléctrica. Con la finalidad de satisfacer las demandas de esta actividad económica, el Estado mexicano desplegó una serie de proyectos hidroeléctricos. En el caso de Valle de Bravo, estado de México, dadas sus características geográficas, geológicas, geomorfológicas e hidrológicas, se impulsó la construcción del sistema hidroeléctrico Miguel Alemán.

Con esto se daría un cambio significativo en el uso de los recursos naturales, pues durante mucho tiempo el espacio donde se ubicó la presa estuvo destinado a la práctica de actividades silvoagrícolas, las cuales constituían la base económica de este lugar, pues se obtenían dos cosechas anuales que eran comercializadas tanto con las poblaciones aledañas como con Toluca y la Ciudad de México. Con la implementación de este proyecto, el Estado dio un nuevo uso al recurso hídrico y expropió la zona 
conocida como «El Plan», lugar donde los campesinos llevaban a cabo sus actividades agrícolas. Para compensar las pérdidas, el Estado dotó a los campesinos de nuevas tierras pero, al encontrarse éstas en zonas con pendientes escarpadas, la práctica de la agricultura produjo escasos beneficios, deteriorándose así la economía de los vallesanos.

Por otra parte, fueron impuestas leyes forestales que impedían el aprovechamiento de los recursos maderables del lugar, pues era indispensable crear una zona boscosa para mantener y atraer la humedad y con ello conservar los niveles de agua del embalse. Esto generó que los pobladores dedicados a otras actividades económicas como la alfarería también se vieran afectados, pues ya no tenían de dónde extraer los minerales ni la leña necesaria para cocerlos.

Estos cambios también tuvieron su manifestación en materia poblacional pues, por una parte, un gran número de habitantes nativos salieron de la cabecera municipal en busca de nuevas fuentes de empleo, pero por otra, los trabajadores que se encargarían de la construcción de las presas formaron nuevos núcleos de población, tal es el caso de Colorines.

En cuanto a los pobladores nativos que se quedaron en el municipio, se vieron en la necesidad de insertarse en las actividades que surgieron del proyecto trabajando como peones, albañiles, cargadores, veladores y otros. Pasaron de ser dueños de sus medios de producción a ser simplemente asalariados.

Por otra parte, la infraestructura creada para la producción de electricidad también conformó un paisaje atractivo caracterizado por un cuerpo de agua rodeado de zonas boscosas, lo que en una etapa posterior permitió la realización de actividades acuáticas que hasta ese momento eran casi imposibles de practicar en el estado de México. Una nueva política territorial de desarrollo facilitaba la llegada de capitalistas fraccionadores ajenos al lugar, motivados también por los incentivos que el Estado otorgó durante ese periodo no sólo al establecimiento de la actividad industrial, sino también al de la actividad turística (García 1979: 18), creándose así nuevos destinos turísticos regionales 
como en el caso de Valle de Bravo.

De esta forma, podemos concluir que, cada vez con más frecuencia, los campesinos de todo el mundo son forzados a sucumbir frente a las presiones e impactos que les son impuestos por las sociedades occidentales en el trayecto hacia el desarrollo económico y la liberación de los mercados. «Los gobiernos de las naciones industrializadas, conjuntamente con las corporaciones transnacionales, han adquirido un apetito insaciable por nuevos suministros de recursos y han acelerado las tasas de extracción de recursos naturales hasta niveles asombrosos» (Sallenave 1992: 41). Sin embargo, esta aproximación al desarrollo trae consigo altos costos sociales y ecológicos, sobre todo para los grupos que dependen para su subsistencia de la preservación de su forma de vida tradicional y de los ecosistemas. Asimismo, las políticas de planeación territorial no constituyen un hecho aislado, sino que más bien son un instrumento que, paralelamente a los cambios en el Estado, van moldeando las estructuras para beneficio del mercado. 


\section{BIBLIOGRAFÍA}

Bernal, Edith, 2011, «Transformación del paisaje natural y cultural de Valle de Bravo», Tesis de maestría, UAEM, México.

Chacón, Orlando, 2004, «El valor estratégico de la Cuenca de Valle de BravoAmanalco, estado de México, como factor para la incorporación regional y global», en Actas Latinoamericanas de Varsovia, v. 27, pp. 121-128.

Código Agrario, 1942, México.

Departamento Agrario, 1954, Decreto que expropia los terrenos del ejido de San Antonio de la Laguna, en Donato Guerra, Méx. Publicado en el Diario Oficial de la Federación, 30 de abril.

—_, 1947, Resolución en el expediente de conflicto comunal entre los pobladores de la cabecera y sus barrios Santa María Ahuacatlán, Otumba y La Peña y el de Acatitlán, Estado de México, 30 de abril.

García, Ana, 1979, Turismo y Subdesarrollo Regional, Universidad Nacional Autónoma de México, México.

McMahon, David, 1973, Antropología de una presa, Instituto Nacional Indigenista, México.

Navarrete, Rosa, 2008, Colorines: Puerta del Sur, Gobierno del Estado de México, México.

Ordenamiento Ecológico, 2002, Ordenamiento ecológico de la cuenca de Valle de Bravo-Amanalco, Tomo I: Diagnóstico, Tomo II: Modelo de ordenamiento, UAEM, Gobierno del Estado de México, Secretaria de Ecología, México. 
Palerm, Ángel, 1993, Planificación Regional y Reforma Agraria, Gernika, México.

Plan Municipal de Desarrollo Urbano de Valle de Bravo (PMDU-VB), 2006, Gobierno del Estado de México, México.

Preston, W., 1999, Una introducción a la teoría del desarrollo, Siglo Veintiuno, México.

Romero Contreras, Tonatiuh, 2000, «Origen y desarrollo de la geografía cultural», en Espacio Geográfico, n. 1. Facultad de Geografía, UAEM, México, pp.7-39.

Sallenave, John, 1992, «El proyecto hidroeléctrico de la Bahía de James: la ruina de un territorio», en Alteridades, n. 4, UAM-Iztapalapa, México, pp. 41-50.

Secretaría de la Economía Nacional, 1946, Decreto que expropia terrenos comprendidos en la curva de embalse a la cota 1833, que se inundará por la presa Valle de Bravo, en el Estado de México. Publicado en el Diario Oficial de la Federación, 19 de noviembre, México

Sierra López, Nancy; Lilia Zizumbo Villarreal, Tonatiuh Romero Contreras y Neptalí Monterroso Salvatierra, 2011, «Ordenamiento territorial, turismo y ambiente en Valle de Bravo, México», en Cuadernos Geográficos, 48 (2011-1), Universidad de Granada, pp. 233-250.

Viqueira, Carmen, 2001, El enfoque regional en antropología, Universidad Iberoamericana. México.

Fecha de recepción: 8 de noviembre de 2010.

Fecha de aceptación: 25 de julio de 2011. 